though active peristalsis is obviously to be avoided. An attempt to carry out some of these principles is contained in the following suggestions, which for the sake of brevity will not include any reference to diet and to nursing, as these matters are now receiving greater attention and are better understood.

The postural treatment. - Often in the course of an enterio attack there is obvious fulness and tension and extensive dulness over the right iliac fossa, and a sensation of weight will be conveyed to the hand when placed behind the loin, whilst the left iliac fossa is soft to the touch and resonant. Now it will be found that if the patient be placed for a few minutes on his left side and once more examined in the supine posture the conditions are entirely reversed, the right flank being resonant and soft and the left iliac fossa or the descending colon dull and resistant. Repeated observation of this result has for the last three years led me to arrange the bed so as to keep the patient at intervals, or in some cases permanently, in a position of slight inclination towards the left. $A$ partial. lateral decubitus which I have usually found to be sufficient for the purpose may easily be obtained by placing a bolster or a firm pillow under the right side of the mattress so that the body is propped over towards the left. A better method, which has the advantage of not disturbing the patient, consists in inclining the bedstead by blocks of suitable beight placed under its two right feet, whilst the patient maintains the dorsal decubitus. Inasmuch as a slight elevation of the foot of the bed is a decided help, a double inclination may be obtained by using three blocks of uneven heights. The bed in this case looks uncomfortable but $I$ have not heard of any complaint on the part of the patient. Either of these methods will fulfil an indication of the first importance by emptying the cæcal pool which I regard as an obstruction and as responsible for fæcal stasis higher up and for the toxic typhoid state. When the first method is resorted to there is no object in its being enforced continuously so long as the state of the bowel is carefully watched and the cæcum and lower ileum are kept empty. In anxious cases the other plan is decidedly to be preferred and should be kept up during the period of danger. The methods in question are, in my experience, free from risk and from appreciable discomfort and I have not been led to suspect that any undesirable strain is thrown upon the diseased bowel.

The medicinal treatment. - The remaining indications have appeared to me to be best carried out by the treatment recommended some years ago by $\mathrm{Mr}$. W. B. Wedgwood of King's Lynn. Though it will not avail against the fatality of the worst attacks it has, so far as I have been able to judge, mitigated the severity of the great majority of cases, and has almost always led to an early amelioration manifested by the disappearance of sordes, the cleaning of the tongue, the clearing of the complexion, and, above all, the return of the appetite. For an adult 20 minims of the liquor hydrargyri perchloridi and from 15 to 20 minims of the tinctura ferri perchloridi with one drachm of syrup of orange or lemon and an ounce of water are administered every six hours throughont the attack, and for 10 days after defervescence to obviate a relapse. Diarrhcea, if present, is quickly stopped, except in the worst cases, and the constipation which often ensues and coincides with an excellent progress is remedied by daily injections of glycerine. The results have been so satisfactory that the administration of alcohol is hardly ever necessary and is carefully avoided lest it should dull the appetite or burden the liver, but port wine is invariably given during convalescence. In the now considerable series of cases treated in this way salivation has never been observed, and the treatment is always well tolerated provided the dose is administered at an interval from milk meals, even should, as is my invariable rule, the milk be peptonised.

To which of the ingredients the greater share of usefulness belongs it would be hard to say. In my opinion they are both valuable. An astringent effect of the iron upon the entire intestine is rendered probable by its constipating effect upon the lower bowel. We may, therefore, reasonably assume that it acts as a local application to the ulcers, whilst it tends to constrict the bowel and its blood-vessels against the risk of bæmorrbage. The many-sided virtues of mercury are probably exerted upon the hepatic, the lymphatir, and the mucous systems, and it is not unwarrantable to suppose that its prolonged administration in frequently repeated doses may be an element in the disinfection of the alimentary canal.

Curzon-street, $\mathbf{W}$.

\section{THE TREATMENT OF A CASE OF ACUTE DILATATION OF THE STOMACH.}

\section{By GEORGE COATES, M.A., M.D. Oxon.}

THE following case of acute dilatation of the stomach in an infant, three weeks old, who finally recovered and in whom the recovery seemed to be entirely due to systematic washing out and cleansing the stomach, appears to be worth recording. I have called the case one of acute dilatation of the stomach, as that was the main feature, though from the collection of cases of hypertrophic pyloric stenosis in infancy in a paper by Dr. Eric Pritchard ${ }^{1}$ I have no doubt that stenosis of the pylorus, either spasmodic or structural, was the cause of the dilatation.

The patient, a girl, was born on Jan. 24tb, 1900, and weighed $7 \mathrm{lb} .13 \mathrm{oz}$ She seemed quite healthy and apparently remained so for nearly three weeks. Her mother endeavoured to nurse her, but failed after trying to do so for some days. She was then fed on humanised milk, from a dairy rompany. On Feb. 14th she began to be frequently sick. From the 17th to the 19th the vomiting suddenly changed its character: she was sick only once in the 24 hours, but then, the nurse said, a very large quantity, at least a pint, came away. soaking all her clothes. At the same time her micturition became very scanty and the bowel contained only small quantities of mucus stained almost black with altered bile. Her stomach was manifestly enormously dilated, whilst from the absence of residue of food in the bowel and the very scanty micturition it was evident that little or nothing passed through the pylorus; but whether there was stenosis of that openirg or whether the enormous dilatation of the stomach had produced there such a kink that nothing could pass through it was not evident. The treatment devised was to feed her at intervals of from two to three hours with small measured quantities of food, and one teaspoonful of the following mixture was given after each meal to aid digestion and to try to prevent fermentation from taking place in the dilated stomach : acidi hydrochlor. dil., 25 minims ; glycerini pepsini acidi, one and a balf drachms; acidi carbolici purissimi, half a grain; aq ad one and a half ounces. On Feb. 19th Dr. Thomas Barlow saw her with me and confirmed the diagnosis and treatment. On the 28th, as she was flowly but steadily losing ground, nutritive enemata were given; each enema consisted of one ounce of peptonised human milk with two drops of brandy. These enemata were given every three hours until the end of May and, with the exception of a few days when there was some diarrhoea, they were always retained and were absorbed. The bowel was washed out once a day with soap and water. Early in March she bad a severe attack of gastro-intestinal catarrh with sickness and diarrbœa. The vomit contained greenish-yellow muco-pus mixed with curds. The stools were frequent and foul-smelling. She bad fever. the temperature varying from $99^{\circ} \mathrm{F}$. in the morning to $102^{\circ}$ at night, and a loud endocardial mitral regurgitant murmur was developed. She lost flesh very rapidly, her weight being $5 \mathrm{lb}, 13 \mathrm{oz}$. on March $17 \mathrm{th}-\mathrm{a}$ loss of $2 \mathrm{lb}$. since birth. Her condition at this time looked perfectly hopeless. She was in a state of extreme emaciation, with shrivelled dry skin and a distended abdomen. The front abdominal wall was so thin that the liver area could be distinctly recognised from the dark colour of that organ showing through it. The dilated stomach could also be easily made out, and slowly moving peristaltic waves were seen passing over it from time to time. For a few days milk was entirely stopped by the mouth and she was put on raw meat, meat juice, and warm water as food, and sulpho-carbolate of soda and carbonate of bismuth as medicine A few days later, as her temperature never rose above $99^{\circ}$ and the diarrhoea had stopped, human milk was given as well. On March 19th she had still lost weight, being now $5 \mathrm{lb}$. $11 \mathrm{oz}$. at seven weeks from birth, when she ought to have weighed over $10 \mathrm{lb}$. From her constant loss of weight and general condition it was cbrious that she must die unless the state 
of her stomach could be improved, and as a last resource it was determined to wash it out with a solution of sodium bicarbonate, 25 grains; sodium sulpho-carbolate, 15 grains ; euthymol, half a drachm; aq. ad one and half ounces. On March 22nd a No. 12 soft elastic catheter, indiarubber tube, and glass funnel were used; the catheter was passed through the month every morning about three hours after a meal. When the catheter entered the stomach some wind would generally escape and then from three to four ounces of thick slime and curdled milk would be evacuated through it. The stomach was then washed out and the resultant flaids were measured. Immediately after the stomach was cleansed a teaspoonful of the following mixture was given: spiritus ætheris chlorici, 15 minims; bismuth carbonate, 40 grains; sodium bicarbonate, 12 grains; :sodium sulpho-carbolate, half a drachm; decoctum hæmatoxyli, six drachms ; aq. cinnamom. ad one and a half ounces ; and a quarter of an hour later she was fed with from two to three ounces of human milk freshly drawn off. She was fed alternately from every two to three hours with from two to three ounces of human milk and one ounce of meat juice. She was not put to the breast as it was considered important that she should have no opportunity of overfilling the stomach. From this time her improvement was decided ; she spent very comfortable days but used to get rather restless by evening and was decidedly uncomfortable during the night. The day after the washing out was commenced she began to pass much more urine; three days later and subsequently she had almost normal motions. At the end of March the meat juice was stopped and she was put regularly to the breast. By April 2nd she had gained two ounces. By the 6th she had gained two more ounces. As her nights were so bad and the days were so good she was from this time washed out regularly night and morning at about three hours after ameal. Each time about three ounces of slime and curd were removed. On the 16 th she had improved so much that a trial was made to see if wash. ing out in the morning only would do, but her nights became restless again, and the evening washing-out of the stomach was resumed. By May 1st she had gained over one pound, her weight being $6 \mathrm{lb} 13 \mathrm{oz}$ She made uninterrapted progress, so that by the 12th the residue of slime and curd remored was reduced to between one and a half and two ounces. After the 19th she was only washed out occa. sionally. On the $23 \mathrm{rd}$, after 48 hours' interval, only one and a half ounces of residue were found. On the $30 \mathrm{ch}$ her weight was $8 \mathrm{lb} .4 \mathrm{oz}$. She had improved steadily ever since and on July 17 th she weighed over $12 \mathrm{lb}$. Her heart was now perfectly normal, the murmur having disappeared on March 29th.

There were two points that are worth noticing: 1. The patient's sensations of hunger were apparently cansed by the absence of digestible food in the stomach. She always showed signs of hunger two or three hours after feeding, even though her stomach, as ascertained by the catheter, might contain three or four ounces of slime and curd. After this had been removed, the stomach cleansed, and two or three ounces of milk given, she would appear to be satisfied for over two hours, although the stomach really contained less material than when she was very hungry. Her sensations of repletion depended on the nutritive value rather than on the quantity of the matter in the stomach. 2. Her attack of severe febrile muco-purulent gastrointestinal catarrh was rapidly followed by the appearance of a loud mitral systolic murmur which resembled in every way one due to rheumatic endocarditis. Soon after the stomach was brought into a healthier condition and the catarrh and fever had subsided, the murmur disappeared, and the heart has since then appeared to be healthy.

Cases of febrile disturbance, accompanied by some gastrointestinal trouble and followed by excited action of the heart and the development of an endocardial murmur, usually at the mitral orifice, are very common in children, and this case appears to show that the endocardill infection may have entered the body from the alimentary canal. It is always dangerous to generalise from only one case, but this one shows that an extremely severe attack of acute dilatation of the stomach was cured by syetematically cleansing that organ twice a day. As the treatment was persevered with the residue of slime and curd became less, and though at first from day to day no great difference could be seen, after every few days it was clear that the food given was being better digested, that less slime was secreted, and that the stomach was in a healthier condition and was regaining its normal functions.

The main point in the treatment appears to be the removal of the residues of slime and curd. This is analogous to the removal of residual urine and mucus in cases of aggravated catarrh of the bladder where the prostate is much enlarged. These residual fluids kept at the temperature of the body form most excellent incubating media for various species of bacteria and promptly infect and contaminate any fresh liquids, whether milk or urine, added to them. Drawing off the residual fluid and washing out the stomach in this case were quite as satisfactory in their results as emptying and washing out the bladder are in these cases of cystitis. Here I may mention that there was some difficulty in getting the catheter to pass the larynx and to enter the oesophagus, but with a little patience it could always be done. Towards the end of the treatment, when the stomach had been restored to a healthy condition, it was evident that it had regained its power of emptying itself through the pylorus. The child had regular natural stools and since then has shown no signs of having an abnormally large stomach or narrow pylorus. I have to thank Dr. Barlow for his valuable aid in February and March.

Brechin-place, S.W.

\section{A CASE OF COMPLETE TRANSVERSE SEPTUM OF THE VAGINA IMPEDING DELIVERY ; URETHRAL COITUS.}

\section{BY E. RUMLEY-DAWSON, L.R.C.P. LOND., M.R.C.S. ENG.}

THE patient was a primipara, aged 21 years, to whom I was called when in strong labour. Her history was as follows. She had had scarlet fever when she was six years of age, but her mother had never mentioned that the attack was in any way unusual, neither had she any recollection of pain or discomfort on sitting down or on micturition. She had never received any injury in the genital region. She had always been regular in her menstrual periods and they were not excessively or abnormally painful. She had never had any symptoms to lead her to think that there was anything abnormal in the vagina. She always had some leucorrhoa but had not seen a medical man for it. The nurse noticing that in spite of strong labour pains no progress seemed to be made at last sent for me.

On examining the patient in the left lateral position the index finger unknowingly and without pain passed direct into the bladder. On more deliberate and ocular examina. tion the external genitals were quite normal but the urethral orifice was found to be very dilated, while the vaginal orifice was small. The finger passed less than an inch into the vagina, meeting with an obstruction which, when a labour pain set in, felt like a large "bag of waters," and bulged with the pain. No cervix could be found and no fornices. The child's head could be felt per vaginam to be pushing down on to the obstruction and making it bulge. On examining per vaginam and rectum it was quite easy to get the obstruction between the fingers. It was found to be a rather thick transverse septum or diaphragm; this could also be plainly felt on examination per urethram and raginam. The septum between the finger-tips was felt to be about one quarter of an inch thick at the insertion into the vaginal wall, thinning down to the centre and becoming thick again at the opposite vaginal insertion. It was evident that a complete transverse vaginal septum was preventing the descent of the head. No hole or aperture could be found anywhere in the septum, but no examination under an anæsthetic was made. The bulging down of the septum by the advancing head so stretched it that even a small hole would have been revealed. The septum was not in the site of the hymen.

Though the patient denied being aware of any abnormality, sexual intercourse it was admitted had been diffcult from the first; while several months after marriage it became impossible, still later there had been less difficulty as the urethra was gradually dilated. The dilatation of the urethra had followed as the result of repeated attempts at coitus; she had not, however, been troubled with incontinence of urine, though urination had become more frequent. This increased frequency may bave been partly 